\title{
Enfermedad de Kawasaki, casos diagnosticados en dos hospitales pediátricos en México de enero de 2004 a marzo de 2020
}

\author{
Kawasaki disease, cases diagnosed in two pediatric hospitals \\ in Mexico from January 2004 to March 2020
}

\author{
Miguel García-Domínguez, ,„ José Quibrera, ${ }^{\S}$ Saúl Canizales-Muñoz," Mariel López-Nogueda," \\ Ángel Rito León-Ramírez, ${ }^{* *}$ Carlos Alberto Velázquez-Ríos, ${ }^{\ddagger, * \star}$ Juan Manuel Carreón-Guerrero, ${ }^{* *}$ \\ Eduardo Llausás-Magaña, ${ }^{\star *}$ Víctor Manuel Pérez-Pico, ${ }^{\star \star} A l e j a n d r o ~ L l a u s a s-V a r g a s{ }^{\star \star, \neq \ddagger}$ \\ * Hospital Pediátrico de Sinaloa. Departamento de Alergia e Inmunología. \\ ‡ Hospital Regional «Dr Manuel Cárdenas de la Vega» ISSSTE. Departamento de Pediatría. \\ $\S$ Hospital Pediátrico de Sinaloa. Departamento de Cardiología. \\ " Hospital Pediátrico de Sinaloa. Departamento de Investigación. \\ " Hospital Pediátrico de Sinaloa. Departamento de Reumatología. \\ ** Hospital Pediátrico de Sinaloa. Departamento de Infectología. \\ \#Centro de Investigación Aplicada a la Salud Publica, Universidad Autónoma de Sinaloa.
}

Culiacán, Sinaloa, México.

\begin{abstract}
Citar como: García-Domínguez M, Quibrera J, Canizales-Muñoz S, López-Nogueda M, León-Ramírez ÁR,
Velázquez-Ríos CA et al. Enfermedad de Kawasaki, casos diagnosticados en dos hospitales pediátricos en México de enero de 2004 a marzo de 2020. Alergia Asma Inmunol Pediatr. 2021; 30 (2): 37-42. https://dx.doi.org/10.35366/101640
\end{abstract}

\section{RESUMEN}

Introducción: La enfermedad de Kawasaki (EK) es una vasculitis de pequeños y medianos vasos, que afecta principalmente a menores de cinco años de edad; su etiología es desconocida, se caracteriza por un síndrome febril agudo con manifestaciones mucocutáneas y puede desarrollar anormalidades de las arterias coronarias (AAC). El tratamiento con inmunoglobulina intravenosa (IGIV) y aspirina previene las AAC y otras complicaciones. Objetivo: Describir las características epidemiológicas, clínicas, tratamiento y complicaciones de la EK en dos hospitales pediátricos en el noroeste del país a lo largo de 16 años. Material y métodos: Estudio retrospectivo de pacientes con EK en dos centros pediátricos. Se obtuvo la frecuencia de los pacientes con diagnóstico de EK completo y EK incompleto. Los resultados se representaron como media, desviación estándar y mediana, según la variable correspondiente. Resultados: Un total de 62 pacientes con EK, el sexo masculino fue de $64.5 \%$, la mediana de edad a diagnóstico de 33.4 meses. La fiebre, rash y conjuntivitis se

\section{ABSTRACT}

Introduction: Kawasaki disease (KD) is a small and medium vessel vasculitis, that mainly affects patients under 5 years age, of unknown etiology, characterized by an acute febrile syndrome with mucocutaneous manifestations and develop coronary artery abnormalities (CAA). Treatment with Intravenous immunoglobulin (IVIG) and aspirin prevent to CAA and other complications. Objective: To known the epidemiological, clinical characteristics, treatment and complications of $K D$ in two pediatric centers in the northwest of the Mexico in the course of 16 years. Material and methods: Retrospective study of patients with KD in two pediatric centers. The frecuency of patients with a diagnosis of complete and incomplete $K D$ was obtained. The results were represented as mean, standard desviation and median, according to corresponding variable. Results: We report 62 patients with $K D$. Males were $64.5 \%$, the median age at the time of diagnosis were 33.4 months. Predominant clinical manifestations were fever, rash and conjunctivitis in more than $86 \%$ of cases. Incomplete $K D$ 
presentaron en más de $86 \%$ de los casos. La EK incompleta fue de $24 \%$. Las anormalidades de las arterias coronarias se presentaron en $29 \%$ de los casos. La presencia de anemia, elevación de proteína C reactiva (PCR) y velocidad de sedimentación globular (VSG) fueron los biomarcadores más relevantes. Todos fueron tratados con IGIV y la resistencia a IGIV fue de $19 \%$. No hubo fallecimientos. Conclusiones: La EK ha mostrado un incremento en los últimos años en México, siendo importante conocer el comportamiento epidemiológico, clínico, tratamiento y complicaciones de EK. Con el surgimiento de la pandemia por COVID-19 se ha observado un incremento de casos de EK, conocidos como síndrome inflamatorio multisistémico pediátrico asociado a SARS-CoV-2.

Palabras clave: Enfermedad de Kawasaki, inmunoglobulina intravenosa, aneurismas coronarios, vasculitis. were $24 \%$. CAA ocurred in $29 \%$ of cases. Anemia, and elevated $C$-reactive protein (CRP) and erythrocyte sedimentation (ESR) were biomarkers relevant. All recived IVIG, the resistance to IVIG were 19\% and there were no deaths. Conclusions: $K D$ has been increasing in recent years of Mexico, being important to known the epidemiological, clinical, treatment and complications. The emergence of COVID-19 pandemic, an increased in KD cases and like-KD, known as multisystem inflammatory syndrome in children associated of SARS-CoV-2.

Keywords: Kawasaki disease, intravenous immunoglobulin, coronary artery aneurysm, vasculitis.

\section{INTRODUCCIÓN}

La enfermedad de Kawasaki (EK) es una vasculitis aguda de pequeños y medianos vasos, autolimitada, de etiología desconocida que afecta principalmente a menores de cinco años y se considera la primera causa de cardiopatía adquirida. ${ }^{1}$

Fue descrita en 1967 por Tomisaku Kawasaki como síndrome de ganglio linfático mucocutáneo febril agudo en 50 niños, manifestado como un síndrome febril de curso agudo y estableciendo los criterios clínicos que se usan hasta la actualidad. ${ }^{2}$

La tasa de mayor incidencia se reporta en Japón con 359, Corea del Sur de 134.4, y EUA de 17.5 a 20.8 por cada 100,000 niños de 0 a 4 años, respectivamente. ${ }^{3,4}$

En México no existen registros de la incidencia y prevalencia de la EK. La mayoría de los centros pediátricos en México ha atendido pacientes con EK; sin embargo, no se ha establecido una red nacional que permita tener datos epidemiológicos confiables como sucede en otros países como Japón y EUA.

Desde que se describió el primer caso en México de EK en 1977 hasta 2012, una revisión de las publicaciones de diversas zonas del país sumaba un total de 250 pacientes. ${ }^{5,6}$

En un intento por tener un registro de la incidencia real de la EK en México a partir de junio de 2012 se estableció un equipo de trabajo denominado: Red de Vigilancia y Prevención de $\mathrm{EK}_{;}^{7}$ sin embargo, hasta la fecha no se ha publicado una actualización de la información.

Posteriormente los dos grandes centros pediátricos en México, el Instituto Nacional de Pediatría (INP) con un total de 687 pacientes hasta 2019 y el Hospital Infantil de México (HIM) con 204 pacientes hasta 2016 han publicado su experiencia con la EK, analizando el comportamiento de la enfermedad, hallazgos de laboratorio, frecuencia de lesiones coronarias, tratamiento y complicaciones en población mexicana. ${ }^{8,9}$
En la actualidad el Centro de Investigación de EK de la Universidad de California en San Diego y la Sociedad Latinoamericana de Infectología Pediátrica crearon la Red de Enfermedad de Kawasaki en América Latina (REKAMLATINA) a partir de 2013 para determinar la incidencia, prevalencia y hallazgos de la enfermedad cardiaca en población latinoamericana, de la cual diversos centros del país forman parte. ${ }^{10}$

Este estudio describe las características epidemiológicas, clínicas, hallazgos de laboratorio, tratamiento y complicaciones de la EK de dos centros pediátricos en el noroeste del país, atendidos a lo largo de 16 años y se hace la comparación con los centros mexicanos que han reportado su experiencia con EK.

\section{MATERIAL Y MÉTODOS}

Se realizó un estudio retrospectivo en un periodo comprendido de marzo de 2004 a marzo de 2020 de dos centros hospitalarios, el Hospital Pediátrico de Sinaloa (HPS) y del Hospital Regional ISSSTE Culiacán, que incluyó pacientes menores de 18 años. El diagnóstico de EK completo se realizó con los criterios establecidos por la American Heart Association (AHA) de 2004 actualizados en 2017. Un criterio mayor: fiebre > cinco días y cuatro de cinco criterios menores: 1. Conjuntivitis bilateral no supurativa; 2. Cambios en labios y mucosa oral; 3. Eritema polimorfo; 4. Adenopatía cervical; y 5. Edema o eritema palmoplantar. Una forma incompleta de EK se definió con la fiebre, dos o tres de los criterios y/o la existencia de anormalidades coronarias. El síndrome de choque por enfermedad de Kawasaki (SCEK), definido como EK que desarrolla hipotensión arterial sistólica para la edad, disminución sostenida $>20 \%$ de la presión arterial sistólica, signos clínicos de hipoperfusión tisular y cambios en el estado mental no considerados por otras condiciones. ${ }^{11,12}$

Se recopilaron datos de los expedientes clínicos en físico y formato electrónico. Se excluyeron los expedientes 
Tabla 1: Manifestaciones clínicas de los pacientes.

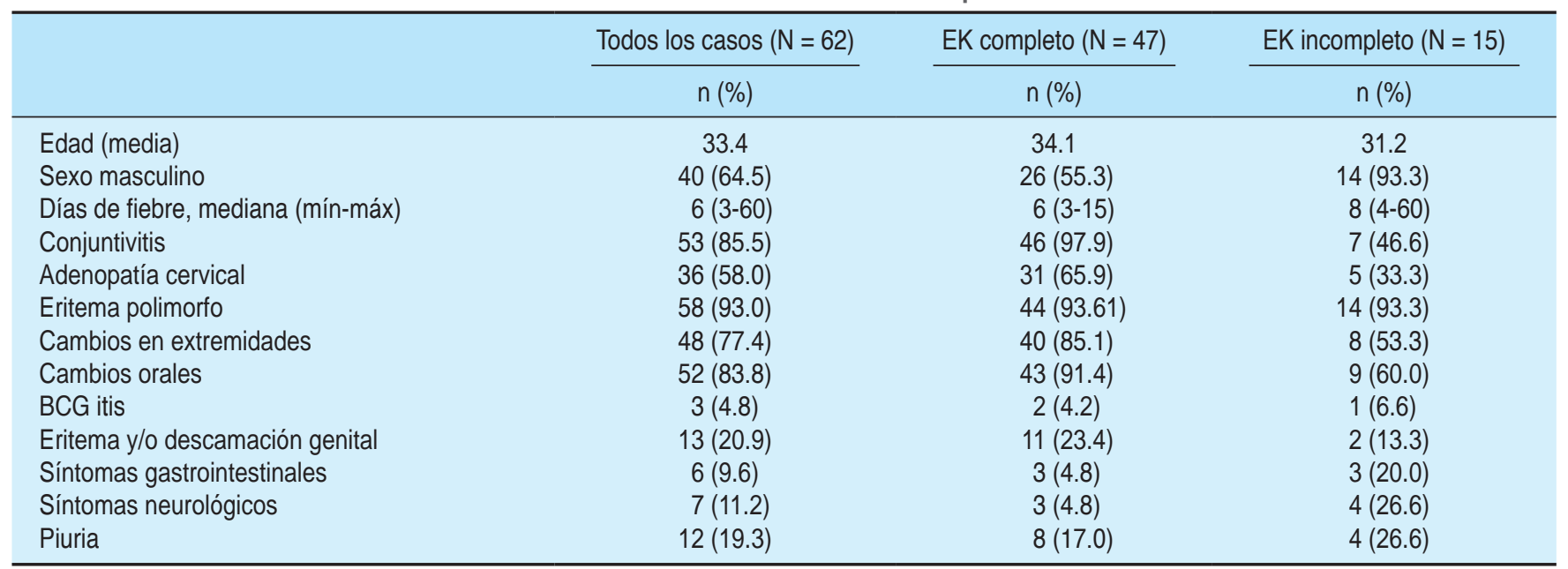

EK = enfermedad de Kawasaki; BCG = Bacilo de Calmette-Guérin.

incompletos, ilegibles y los que cursaron con enfermedades infecciosas concomitantes en las dos semanas previas a la aparición de los síntomas. Los pacientes fueron tratados con inmunoglobulina intravenosa (IGIV) $2 \mathrm{~g} / \mathrm{kg} /$ dosis única, ácido acetil salicílico de $80-100$ mg/kg/día y a partir de 2017 a $30-50 \mathrm{mg} / \mathrm{kg} /$ día, los esteroides se utilizaron en pacientes con riesgo de desarrollar lesiones coronarias o resistencia a IGIV, esta última se consideró ante fiebre persistente mayor de 36 horas de la finalización de la primera dosis de IGIV.

Se recolectaron las características clínicas, hallazgos de laboratorio, tratamiento, complicaciones y desarrollo de anomalías de arterias coronarias (AAC). Para el análisis de datos se utilizó el programa Statistical Product for the Social Science (SPSS) versión 23. Los resultados se representaron como media, desviación estándar y mediana según corresponde a la variable.

\section{RESULTADOS}

En total se analizaron 62 casos en el periodo de estudio. A partir de 230,773 atenciones en el periodo correspondiente se calculó la prevalencia de la EK en el HPS con 48 pacientes, en $0.021 \%$ (IC 95\% de 0.020-0.034\%).

El sexo masculino fue de $64.5 \%$ con una mediana de edad de 33.4 meses de edad (3-96 meses), con una mediana de los días de fiebre de seis días. De los pacientes, $24 \%$ presentaron EK incompleta. Los signos y síntomas se encontraron en orden de frecuencia: fiebre 100\%, eritema polimorfo $93 \%$, conjuntivitis $85.5 \%$, cambios en la mucosa oral $83.8 \%$ y cambios en las extremidades $77.4 \%$. Otras manifestaciones relevantes fueron eritema de la zona genital $20.9 \%$, reacción en la zona de aplicación de vacuna BCG (bacilo de Calmette-Guérin) $4.8 \%$ y piuria en $19.3 \%$ (Tabla 1). Se observó predominio estacional en primavera (35.5\%).
Los estudios de laboratorio relevantes observados fueron anemia, velocidad de sedimentación globular (VSG) y proteína $C$ reactiva (PCR) elevadas (Tabla 2).

Las anormalidades coronarias se encontraron en $29 \%$ de los pacientes, ectasia en $27.4 \%$, aneurismas coronarios en $19.3 \%$ de los casos y aneurismas gigantes en $1.6 \%$ (Tabla 3).

Las diferencias entre las formas incompletas de las completas fueron predominio del sexo masculino (93.3\% vs. $55.3 \%$ ), dos días más de fiebre al diagnóstico, síntomas gastrointestinales ( $20 \%$ vs. $4.8 \%$ ), síntomas neurológicos (26.6\% vs. 4.8-\%), en los hallazgos de laboratorio se observó mayor recuento plaquetario, PCR y VSG.

De los pacientes, $100 \%$ recibió dosis de IGIV, 95\% a dosis de $2 \mathrm{~g} / \mathrm{kg} / \mathrm{do}$, y $5 \%$ entre 1 y $1.9 \mathrm{~g} / \mathrm{kg} / \mathrm{do}$ en dosis única, la refractariedad a IGIV se observó en 12 pacientes (19.3\%) y el uso de esteroide fue de $8.7 \%$. Se suministró aspirina a $100 \%$ de los pacientes en un rango de 30 a $100 \mathrm{mg} / \mathrm{kg} /$ día y posteriormente de 3 a $5 \mathrm{mg} / \mathrm{kg} /$ día. Dos pacientes (3.2\%) cumplieron con criterios de síndrome de choque por enfermedad de Kawasaki (SCEK). Sólo un paciente recibió pulso de metilprednisolona por SCEK dosis única. No se usaron biológicos en ninguno de los pacientes. No hubo fallecimientos.

\section{DISCUSIÓN}

La EK ha ido en incremento en diversos hospitales pediátricos en México. El INP es el hospital que más casos tiene registrados y demuestra en diferentes periodos cómo han incrementado los casos nuevos de EK: con 48 casos de 1995 a 2003; 108 casos de 2004 a 2008; 182 casos de 2009 a 2013; 170 casos de 2014 a 2016; y 179 casos de 2017 a $2019 .{ }^{8,13,14}$ A pesar de que se trata de un único 
centro, podría ser el reflejo de la casuística de los otros centros hospitalarios pediátricos del país que nos han permitido conocer la presentación clínica, afección coronaria, tratamiento y complicaciones..$^{6-8}$ La REKAMLATINA a partir de 2013 permite conocer mayores datos estadísticos en Latinoamérica. ${ }^{10}$

LA EK ha sido reconocida en el HPS desde julio, y la primera publicación de un caso en el año $2008 .{ }^{15}$ Es a partir de marzo de 2004 que se han podido documentar los diagnósticos hasta marzo de 2020, previo a la pandemia de COVID-19, con un total de 62 casos, de los cuales 48 fueron atendidos en el HPS. En la Tabla 4 se desglosan los centros en México que han reportado su experiencia en EK. $6,8,9$

Los datos demográficos generales como edad y sexo son similares a los reportados por centros en México, donde la edad de presentación de nuestro hospital es de 33.4 meses (de 32 a 37 meses en los otros centros), 22.6\% fue menor de 12 meses igual al reportado por el HIM y el INP, con un predomino del sexo masculino en $64.5 \%$, al igual que los demás centros con una relación mayor de 1.5:1 respecto al sexo femenino. $6,8,9$

La presentación de EK incompleta fue de 24\%, discretamente mayor que en los otros centros, lo que puede deberse a la identificación difícil en los primeros casos estudiados. La presencia de aneurismas coronarios fue de $20 \%$, un porcentaje intermedio comparado con los demás centros (de 10 a 36\%). De los pacientes, 96.7\% recibió IGIV a $2 \mathrm{~g} / \mathrm{kg}$ y $19 \%$ requirió una segunda dosis de IGIV, porcentajes mayores que lo reportado en los demás centros; sin embargo, no pudimos determinar algún factor de riesgo asociado en el análisis estadístico.

De las manifestaciones clínicas, la fiebre fue de $100 \%$ al igual que lo reportado por Sotelo-Cruz y colaboradores, y el HIM; ${ }^{6,8,9}$ sin embargo, también se han documentado casos de EK sin fiebre. ${ }^{16}$ El rash cutáneo fue de $93 \%$ como signo más frecuente de los criterios diagnósticos de EK, similar al reportado por Sotelo-Cruz y su equipo, mientras que en el INP es el tercero y en el HIM el cuarto en orden de frecuencia. ${ }^{6,8,9}$ La inyección

Tabla 2: Hallazgos de laboratorio de los pacientes.

\begin{tabular}{lccc}
\hline & Todos los casos $(\mathrm{N}=62)$ & EK completo $(\mathrm{N}=47)$ & EK incompleto $(\mathrm{N}=15)$ \\
\hline Hemoglobina* $^{*}$ & $10.89 \pm 1.36$ & $10.73 \pm 1.09$ & $10.94 \pm 2.03$ \\
Plaquetas $^{* *}$ & $396(65-827)$ & $361(106-827)$ & $506(65-798)$ \\
Neutrófilos $^{*}$ & $12,234.6 \pm 6,252.6$ & $12,340 \pm 5,829$ & $11,894 \pm 7,698$ \\
Linfocitos $^{*}$ & $4,088.6 \pm 3,146.9$ & $3,544 \pm 2,284$ & $5,837 \pm 4,711$ \\
PMN $^{*}$ & $66.6 \pm 15.19$ & $69.2 \pm 11.1$ & $58.3 \pm 22.6$ \\
PCR $^{* *}$ & $10.5(0.05-50.4)$ & $9.14(0.05-45.2)$ & $17.6(1.77-50.4)$ \\
Na $^{*}$ & $136.2 \pm 2.63$ & $135.9 \pm 2.67$ & $137 \pm 2.49$ \\
VSG $^{* *}$ & $50.57(5-532)$ & $49.6(5-532)$ & $55(32-64)$ \\
Albúmina & $3.33 \pm 0.66$ & $3.44 \pm 0.55$ & $3.14 \pm 0.90$ \\
IGIV, $n$ (\%) & $60(96.7)$ & $45(75.0)$ & $15(25.0)$ \\
Segunda dosis de IGIV, $n(\%)$ & $12(19.3)$ & $8(66.7)$ & $4(33.3)$ \\
Esteroides, $n(\%)$ & $4(8.7)$ & $2(5.7)$ & $2(18.2)$ \\
Aspirina, $n(\%)$ & $60(96.7)$ & $45(75.0)$ & $15(25.0)$ \\
\hline
\end{tabular}

Resistencia a GGIV son los que recibieron una segunda dosis de IGIV.

* Media \pm desviación estándar. ** Mediana (mínimo-máximo).

EK = enfermedad de Kawasaki; PMN = polimorfonucleares; $P C R$ = proteína $C$ reactiva; $V S G$ = velocidad de sedimentación globular; IGIV = inmunoglobulina intravenosa; GGIV = gammaglobulina intravenosa .

Tabla 3: Resultados ecocardiográficos al momento del diagnóstico.

\begin{tabular}{|c|c|c|c|}
\hline & Todos los casos $(\mathrm{N}=62)$ & EK completo $(\mathrm{N}=47)$ & EK incompleto $(\mathrm{N}=15)$ \\
\hline & $n(\%)$ & $n(\%)$ & $\mathrm{n}(\%)$ \\
\hline Anormal & $18(29.0)$ & $11(23.4)$ & $7(46.6)$ \\
\hline Ectasia & $17(27.4)$ & $11(23.4)$ & $6(40.0)$ \\
\hline Aneurisma coronario & $12(19.3)$ & $8(17.0)$ & $4(26.6)$ \\
\hline Aneurisma gigante & $1(1.6)$ & 0 & $1(6.6)$ \\
\hline LMCA & $5(8.0)$ & $2(4.2)$ & $3(20.0)$ \\
\hline RCA & $6(9.6)$ & $4(8.5)$ & $2(13.3)$ \\
\hline
\end{tabular}

Por sus siglas en inglés: $\mathrm{LMCA}=$ arteria coronaria izquierda; $\mathrm{RCA}=$ arteria coronaria derecha. 
Tabla 4: Principales características de la EK de las publicaciones en México.

\begin{tabular}{lcccc}
\hline Autor & Sotelo-Cruz $^{6}$ & García-Rodríguez $^{9}$ & Garrido-García $^{8}$ & Casos reportados $^{\circ}$ \\
\hline Pacientes & 250 & 204 & 687 & 62 \\
Edad (meses) & $36-60$ & 32.5 & $37.6 \pm 35.5$ & 33.4 \\
Hombres (\%) & 76.8 & 55.0 & 65.6 & 64.5 \\
Relación Hombre:Mujer & $3.3: 1$ & $1.5: 1$ & $1.91: 1$ & $1.81: 1$ \\
EK incompleto (\%) & 9.2 & 20.0 & 20.2 & 24.1 \\
$<12$ meses (\%) & ND & ND & 22.1 & 22.6 \\
AAC (\%) & ND & 29.4 & ND & 29 \\
Aneurismas coronarios (\%) & 36.0 & 10.8 & 29.6 & 19.3 \\
IGIV (\%) & 78.0 & 82.8 & 92.4 & 96.7 \\
Segunda dosis de IGIV (\%) & 8.8 & 8.8 & 6.5 & 19.3 \\
Esteroides (\%) & 6.0 & 2.9 & 46.8 & 8.7 \\
Hallazgos de laboratorio & Anemia, leucocitosis, neutrofilia, & Leucocitos, PMN, PCR y & Anemia, leucocitosis, & Anemia, VSG y \\
& VSG, PCR y plaquetas $\uparrow$ & VSG $\uparrow$ & neutrofilia & PCR $\uparrow$ \\
Mortalidad (\%) & 1.2 & 1.0 & 0.29 & 0 \\
\hline
\end{tabular}

EK = enfermedad de Kawasaki; ND: no disponible; $A A C$ = aneurismas de arterias coronarias; IVIG = inmunoglobulina intravenosa; VSG = velocidad de sedimentación globular; $\mathrm{PCR}=$ proteína $\mathrm{C}$ reactiva.

conjuntival se presentó en $85.5 \%$, mientras que los demás reportaron de 89 a $90 \%$ de los casos. El tercer signo más frecuente fue el cambio en labios y cavidad oral en $83.8 \%$, mientras que fue el signo más frecuente en lo reportado por el INP (93.3\%). Los cambios en las extremidades se presentaron en $77 \%$, Sotelo-Cruz y colegas reportaron hasta $90 \%$ de los casos. El menos frecuente de los signos coincide en todos los centros entre 55 y $60 \%$ de los casos. ${ }^{6,8,9}$

Otros hallazgos asociados relacionados con la EK se observan en menor frecuencia como los cambios en la zona de aplicación de la vacuna BCG, nuestra serie de casos reporta $4.8 \%$ similar a Sotelo-Cruz y colaboradores, y el HIM; sin embargo, el INP reporta hasta $29.4 \% .^{6,8,9}$ Otro signo relevante en la EK es la descamación de la zona perianal, la cual encontramos en $20.9 \%$ de los casos, mientras que los demás estudios varían entre 10 y $30 \%{ }^{6,8,9}$ Los síntomas gastrointestinales fueron de $9.6 \%$ similar a Sotelo-Cruz y su equipo y el HIM; sin embargo, Gámez-González y su equipo reportaron que $91 \%$ de 11 pacientes que desarrollaron SCEK presentaban compromiso gastrointestinal comparado con aquéllos sin choque. ${ }^{17}$ Esta forma grave de EK no es reportada por Sotelo-Cruz y colegas y el HIM, mientras que el INP representa $4.36 \%$ de los casos. ${ }^{6,8,9}$ En nuestro reporte representa $3.2 \%$ y uno de los casos fue menor de 12 meses, con EK incompleta, con síntomas gastrointestinales y desarrollo de aneurismas coronarios gigantes. ${ }^{18}$ No tuvimos defunciones; sin embargo, éstas se reportan en menos de $1.2 \%$ en los otros centros. ${ }^{6,8,9}$

De 15 a 20\% de los pacientes no tienen los estudios completos al ingreso y estancia hospitalaria, lo que nos impidió identificar asociaciones o factores de riesgo, además de que no fue posible determinar la prevalencia en los 14 pacientes del Hospital Regional del ISSSTE.

\section{CONCLUSIONES}

La EK en México ha ido en incremento en parte debido al aumento en el número de casos, publicaciones de las series y reportes de casos, así como a un incremento en el reconocimiento y juicio clínico de cada centro hospitalario pediátrico. Los centros pediátricos en Sinaloa también han registrado EK desde 1991. Encontramos que la EK se presenta en dos terceras partes de los casos, la edad media al diagnóstico es de 33.4 meses, siendo el rash cutáneo y conjuntivitis no purulenta los signos más frecuentes. La presentación incompleta de EK fue de $24 \%$, anormalidades de las arterias coronarias fue de $29 \%$ y aneurismas coronarios fue de $19.3 \%$ de los casos, hallazgos que son parecidos a los publicados en diferentes centros pediátricos en México.

Es necesario establecer una red de registro nacional de EK y que los centros de atención pediátrica de México que pertenecen a REKAMLATINA den a conocer los datos epidemiológicos para estimar incidencia y prevalencia, diagnóstico, tratamiento y complicaciones de la EK con la finalidad de establecer en el Sistema de Salud que la EK sea reporte de carácter obligatorio, ya que con el surgimiento de la pandemia por COVID-19 se ha observado un incremento en el país y en todo mundo de casos similares a EK, conocido como síndrome inflamatorio multisistémico pediátrico asociado con SARS-CoV-2.

\section{AGRADECIMIENTOS}

Agradecemos al Dr. Marco Antonio Yamazaki Nakashimada del Instituto Nacional de Pediatría por sus valiosas sugerencias y comentarios y a la Dra. Fabiola Ordorica Sandoval en el seguimiento de pacientes del Hospital Regional ISSSTE. 


\section{REFERENCIAS}

1. Gordon JB, Kahn AM, Burns JC. When children with Kawasaki disease grow up: myocardial and vascular complications in adulthood. J Am Coll Cardiol. 2009; 54 (21): 1911-1920. doi: 10.1016/j.jacc.2009.04.102.

2. Kawasaki T, Kosaki F, Okawa S, Shigematsu I, Yanagawa H. A new infantile acute febrile mucocutaneous lymph node syndrome (MLNS) prevailing in Japan. Pediatrics. 1974; 54 (3): 271-276.

3. Ae R, Makino N, Kosami K, Kuwabara M, Matsubara Y, Nakamura Y. Epidemiology, treatments, and cardiac complications in patients with Kawasaki disease: the nationwide survey in Japan, 2017-2018. J Pediatr. 2020; 225: 23-29.e2. doi: 10.1016/j.jpeds.2020.05.034.

4. Lin MT, Wu MH. The global epidemiology of Kawasaki disease: review and future perspectives. Glob Cardiol Sci Pract. 2017; 2017 (3): e201720. doi: 10.21542/gcsp.2017.20.

5. Rodríguez-Suárez S. Síndrome linfomucocutáneo. Bol Med Hosp Infant Mex. 1977; 34: 53-57.

6. Sotelo-Cruz N. Revisión de la Enfermedad de Kawasaki en México, desde la perspectiva de las publicaciones médicas (enero de 1977 a mayo de 2012). Arch Cardiol Mex. 2013; 83 (3): 214-222.

7. Coria LJJ. Enfermedad de Kawasaki. Grupo Red para la Vigilancia y Prevención de Enfermedad de Kawasaki en México. Rev Enfer Infec Pediatr. 2012; 25.26 (102): 213-214.

8. Garrido-García LM, Gaffare-Aranda S, Cravioto P, Galván F, UlloaGutiérrez R, Yamazaki-Nakashimada MA. Enfermedad de Kawasaki en lactantes bajo un año de edad. Un reto para el diagnóstico y tratamiento de los pacientes. Experiencia en un centro hospitalario en México. Rev Chil Infectol. 2020; 37 (5): 584-590. doi: 10.4067/ S0716-10182020000500584.

9. García-Rodríguez F, Flores-Pineda AJ, Villareal-Treviño AV et al. Enfermedad de Kawasaki en un hospital pediátrico en México. Bol Med Hosp Infant Mex. 2016; 73 (3): 166-173. doi: 10.1016/j. bmhimx.2016.01.002.
10. Ulloa-Gutiérrez R, Salgado AP, Tremoulet AH. Kawasaki disease in Latin American children: past, current, and future challenges. J Pediatric Infect Dis Soc. 2014; 3 (4): 280-281. doi: 10.1093/jpids/piu105.

11. McCrindle BW, Rowley AH, Newburger JW, Burns JC, Bolger AF, Gewitz $\mathrm{M}$ et al. Diagnosis, treatment, and long-term management of Kawasaki disease: a scientific statement for health professionals from the American Heart Association. Circulation. 2017; 135 (17): e927-e999. doi: 10.1161/CIR.0000000000000484.

12. Kanegaye JT, Wilder MS, Molkara D, Frazer JR, Pancheri J, Tremoulet $\mathrm{AH}$ et al. Recognition of a Kawasaki disease shock syndrome. Pediatrics. 2009; 123 (5): e783-e789. doi: 10.1542/peds.2008-1871.

13. Garrido-García LM, Soto-Blanquel JL, Espinosa Rosales FJ. Enfermedad de Kawasaki: cuadro clínico, exámenes de laboratorio y lesiones coronarias. Acta Pediatr Mex. 2015; 36: 314-321.

14. Garrido-García LM, Peña-Juárez RA, Yamazaki-Nakashimada MA. Manifestaciones cardiacas en la etapa aguda de la enfermedad de Kawasaki en un hospital pediátrico de tercer nivel en la Ciudad de México. Arch Cardiol Mex. 2018; 88 (5): 441-446 doi: 10.1016/j. acmx.2018.03.005.

15. Pérez-Pico VM, Llausás-Magaña E et al. Enfermedad de Kawasaki en un lactante de 3 meses. Descripción de un caso. Pediatr Mex. 2008; 1 (1): 11-13.

16. Saltigeral Simental P, Garrido García LM, Camacho Reyes L, Soto Amador K, Yamazaki Nakashimada MA. Enfermedad de Kawasaki. La fiebre debe ser un criterio obligatorio? Rev Alerg Mex. 2008; 55 (4): $176-179$.

17. Gámez-González LB, Murata C, Muñoz-Ramírez M, YamazakiNakashimada M. Clinical manifestations associated with Kawasaki disease shock syndrome in Mexican children. Eur J Pediatr. 2013; 172 (3): 337-342. doi: 10.1007/s00431-012-1879-1.

18. García-Domínguez M, Riviera-Navarro D, Quibrera J, PérezGaxiola G. Aneurismas coronarios gigantes en lactante con síndrome de choque por enfermedad de Kawasaki. Rev Alerg Mex. 2020; 67 (2): 174-182 doi: 10.29262/ram.v67i2.737. 